Scheorer u. Drechsel: Künstliche Darstellung etc. 63

\title{
Künstliche Darstellung von Flussspath und Schwerspath;
}

\author{
von \\ Th. Scheerer und E. Drechsel.
}

Fluorcalcium in krystallisirter Gestalt ist, soweit ans bekannt, auf künstlichem Wege bisher nicht elreugt worden. In der gekrönten Preissohrift "die künstlich dargestellten Mineralien" von Prof. Dr. Fuchs (Haarlem 1872) findet man keine hierauf bezügliche Thatsache angeführt. Die wichtige Rolle, welche der Flussspath in vielen, namentlich auch in gewissen Freiberger Kuzgängen spielt, veranlasste uns einige Versuche über künstliche Flussspathbildung vorzunehmen, deren Resultate wir im Folgenden mittheilen.

Sowohl gepulverter Flussspath, als amorphes Fluorcaicium (durch Mischung von Flusssäure mit einem aufgelösten Kalksalz erhalten) zeigen sich bei stärkerer Glühhitze löslich in gewissen geschmolzenen Chlormetallen, besonders in Chlorcalcium, Chlorkalium and Chlornatrium, sowio in Gemischen dioser Salze. Bei derartigen Ziusammenschmelzungen in einem Platintiege] über dem Gasgebläse bildete sich bei möglichst verzögorter Abkïhlung; krystallisirtes Fluorcalcium, welches durch Auskochen der Sehmelze durch Wasser gesondert erhalten warde. Dasselbe bestand, wie die mikroskopische Untersuchung zeigte, grösstentheils aus tesseralen Krystallskeletten, gebildet durch rechtwinklig an einander sefügte $Z$ weige, jeder Zweig aus an und über einauder gewachsenen Oktaëdern bestehend, deren Hauptaxen unter sich und mit den drei Zweigrichtungen parallel liefen; ganz wie solche Gebilde bei andern tesseralen Stoffen bekannt sind, nameutlich beim Kupfer, Silber, Salmiak, Alann. Hier und da waren auch isolirte, ringsum ung gebildete Oktä̈der bemerkbar. Nirgends aber liessen sich flexaêder entdecken, nicht ein. 


\section{Sche日rer u. Drechsel: Künstliche Darstellung}

mal als Combinationen an Oktaëdern. Was uns auf trocknem Wege nicht gelang, Flussspath in seiner gewöhnlichen Krystallform darzustellen, suchten wir jetzt auf nassem Wege - zum Theil mittelst Ueberhitzung unter hohem Dampfdruck - zu erreichen. Abgesehen von einigen missglïckten Versuchen, gelang uns dies in verschiedenem Grade durch folgende Methoden.

Eine Auflösung von saurom Kieselfluorcalcium (bereitet durch Lösen krystallisirten neutralen Kieselfluorcalciums und Abfiltriren des allmählich ansgeschiedenen basischen Salzes) wurde mit etwa dem gleichen Volum einer mässig verdünnten, neutralen Chlorcalciumsolution versetzt. Die gemischte Flüssigkeit, welche einen beträchtlichen UeberHuss an Chlorcalcium im Verhältniss zum Kieselfluorcalcium enthielt, zeigte erst nach einigen Stunden Spuren von Trübung. Sie wurde in einer zugeschmolzenen Glasröhre, unter Anwendung bekannter Vorkehrungen (eines sogrenannten Kanonen-Apparates) während 10 Stunden biz auf eine Temperatur von etwa $250^{\circ}$ erhitzt. Nach dem Erkalten und Oeffnen der Röhre, sowie nach Entfernung der darin enthaltenen Flüssigkeit sammt dem losen - meist aus amorphem Kieselsäurehydrat bestehenden - Niederschlage, wurde die Röhre mit Wasser mehrmals ausgespült und längere Zeit behandelt, um. jeden löslichen Stoff völlig zu entfernell. An der so gereinigten and durch Frwärmung wieder getrockneten Röhrenwandung gewahrte man, selbst schon bei mässiger Vergrösserung mittelst der Loupe, sehr scharf ausgebildete Krystalle. Einige derselben hatten Oktaëder-Form, andere waren Combinationen von Oktaëder und Hexaëder, letztere Gestalt jedoch niemals für sich auftretend. An den grössten dieser Krystalle ereichten die Kanten eine Lünge von $0,07 \mathrm{Mm}$. - Wiederholungen dieses Versuchs gaben anfangs kein so günstiges Resultat; bis sich heraussellte, dass zum vollkommnen Gelingen eine Temperatiur von nicht unter $240^{\circ}$ erforderlich sei. Sohöne und ausserordentlich zahlreiche Krystalle bildeten sich bei einer fast $250^{\circ}$ erreichenden Temperatur. Ein höherer Hitzgrad hatte 
leider stets das Explodiren unserer Glasröhren zur Folge, obwohl sie aus schwer schmel abarem Kaliglas bestanden und $3 \mathrm{Mm}$. Wanddioke hei kaum $14 \mathrm{Mm}$. Durchmesser im Lichten besassen. Dagegen ist es nicht nothwendig, jene filtrirte saure Solution von Kieselfluorealcium anzuwenden, sondern es genügt, krystallisirtes (neutrales) Kieselfuorcalcium in fester Gestalt in die Röhre zu bringen und Chlorcalciumlösung darauf zu giessen. Dann wird die Röhro so zugesehmolzen, dass etwa ein Drittel ihres Innern mit Luft erfüllt bleibt. im Kanonen-Apparat allmählich bis suf $250^{\prime \prime}$ erhitzt, einige Stunden in dieser Temperatur erhalten und darauf langsam abkühlen gelassen. Die gebildeten Flussspathkrystalle sitzen so fest an der ... vollkommen durchsichtig gebliebenen - Röhrenwandung, dass keine Gefahr vorhanden, sie beim wiederholten Ansspülen der Röhre wegzuwaschen. Wir erhielten auf diese Weise so zahlreiche Krystalle, dass die Röhrenwandung stellenweise mit kleinen Oktäidern dicht inkrustirt war. Die meisten derselben hatten etwa 0,02 Mm. Kantenlänge: darunter kamen aber einzelne grössere mit Kantenlëngen bis zu 0,08 Mm. vor. Unzweifelhaft wïrden sich Krystalle von noch bedeutenderer Grösse durch geräumigero und stärkere Glasröhren, durch höhere Temperatur und längere Versuchsdaner erzeugen lassen. Da es uns jedoch ausschliesslich uuf die wissenschaftliche Darlegung der Thatsache ankarn, glaubten wir hiervon absehen zu dürfen.

Der chemische Hergang bei dieser gegenseitigen Zorsetzung der genamnten beiden Salze lässt sich folgendermaassen auffassen.?

$$
\left.\begin{array}{r}
3 \mathrm{CaF}+2 \mathrm{SiF}_{9} \\
6 \mathrm{Ca} \Theta 1 \\
\mathrm{HO}
\end{array}\right\}= \begin{cases}9 \mathrm{CaF} \\
2 \mathrm{SiO} \\
6 \mathrm{Hel}\end{cases}
$$

Als Zersetzungsprodukte werden also Fluorcalcium (Flussspath), Kieselsäure (in Gestalt von Kieselsäurehydrat) und Chlorwasserstoffsäure gebildet.

1) Mit Zugrundelogung der B erzeli us sehen Formel für die Kieselsäure $=\mathrm{SiO}_{\mathrm{g}}$. Durch Annahme yon $\mathrm{SiO}_{2}$ wird hier nichts Weventliches verändert.

Journ. f. prakt. Chemie [2] Bd. 7. 
Eine ähnliche Zersetzung muss stattfinden, wenn man, anstatt des Kieselfuorcalciums, Kieselflusssäure auf Chlorcalcium einwirken lässt.

$$
\left.\begin{array}{rl}
3 \mathrm{HF}+2 & \mathrm{BiF}_{3} \\
9 & \mathrm{Ca} G \mathrm{Gl} \\
6 & \mathrm{HO}
\end{array}\right\}=\left\{\begin{array}{lll}
9 & \mathrm{CaF} \\
2 & \mathrm{SiO}_{3} \\
6 & \mathrm{Hel}
\end{array}\right.
$$

Der Unterschied besteht blos in einer vermehrten Chlorwasserstoffsüure-Bildung. Allein theils diese erböhte Säuremenge, theils die freie Kieselfluorwasserstoffsäure wirken ungünstig auf die Glasröhre and erschweren die Anwendung hoher Temperaturen. Ein derartiger Versuch bei etwa $238^{\circ}$ auggeführt lieferte ein körniges Gebilde ohne deutlich wahrnehmbare Krystalle.

Nachdem es uns auf beschriebene Art gelungen war, Flussepath als Zersetzungsprodukt zu erzengen, versuchten wir Flussspathkrystalle auf einfacherem Wege darzustellen . aus einer Solution von Fluorcalcium in Wasser. Frisch bereitetes amorphes Fluorcalcium ist in Wasser nicht ganz unlöslich. Eine solche Solution gab beim Verdunsten sowohl über Schwefelsäure in Exsiccator als im Vacuum, keine deutlich erkennbaren Krystalle bei 100 facher linearer Vergrösserung, deren wir uns meist bei unseren Versuchen bedienten Selbst bei 600 facher Vergrösserung blieb es ungewiss, ob einige als hexaëdrische Gestalten erscheinende Gebilde wirklich diesen Charakter besassen. Als dagegen aroorphes Fluorcalcium, mit schwach durch Salzaăure angesäuertem Wasser') übergossen, in einer zugeschmolzenen Glasröhre während 10 Stunden bis auf $240^{\circ}$ (einem Dampfdruck von etwa 32 A.tmosphären entsprechend) erhitzt wurde, erhielten wir zahlreiche, sehr scharf ansgebildete Krystalle, allein vur Oktä̈der (meist mit Kantenlängen von $0,02 \mathrm{Mm}$.) an denen Hexaëder-Flächen mit Sicherheit nicht erkannt werden konnten.

1) Fine solche schwache Ansäuerung ist nothwendig, un die Glasröhre gegen das Angreifen durch die Flüssigkeit zu sohützen. Reines Wasser wie neutrale oder gar alkalisoh reagirende Flüsøigkeiten haben stets das Explodiren der Röhren bei höheren Temperaturen zur Folge. 
Fluorbaryum ist dem Fluorcalcium chemisch so verwandt, dass es nahe lag, auch über die krystallinische Darstellung dieser Verbindung einige beiläufige Vorsuche anzustellen.

Durch Zusammenschmelzen vou amorphem Fluorbaryum mit Chlorcalium und Chlornatrium wurden, nach Auslaugung der Schmelze mit Wasser, keine krystallinischen Gebilde bemerkbar. Fluorbaryum mit Fluorkalium zusarnmengeschmolzen, gab wenigstens skelettartige Gebilde, die allenfalls auf tesserale Gestaltung bezogen werden konnten. (Einen entsprechenden Versuch hatten wir frïher beim Fluorcalcium angestellt, doch ebenfalls ohne den gewünschten Erfolg.)

Da Fluorbaryum erheblich weniger schwerlöslich in Wasger ist als Fluorcalcium, so liess sich erwarten, dass mikroskopische Krystalle desselben sich direct würden darstellen lassen. In der That lieferte eine gesättigte Lösung davon bei der Verdunstung im Exsicoator einen krystallinischen Rückstand, in welchem sich einzelne Gruppen von völlig ausgebildeten $\mathrm{Hexaëdern} \mathrm{(mit} \mathrm{Kantenlängen} \mathrm{bis}$ zu $0,02 \mathrm{Mm}$.) unterscheiden liessen. Sie befanden sich vorzugsweise an den senkrechten Wänden des Glases, in welchem die Verdunstung erfolgte, während dessen flacher Boden mit weniger deutlichen krystallinisehen Gebilden bedeckt erschien. - Fluorbaryumlösung mit einem Zusatz von ungelöstem amorphen Fluorbaryum und einigen Tropfen Salzeäure in einer zugeschmolzenen Glasröhre auf $230^{\circ}$ erhitzt, gab eine klare Iösung, aus welcher sich viele kleine prismatische Krystalle, zum Theil in sternförmigen Gruppen abgesetzt hatten. Nach der damit vorgenommenen chemischen Prüfung, soweit diese mit so geringem Material möglich war, bestanden dieselben aus Fluorbaryum und Chlorbaryum. Bei einem z,weiten Versuche dieser Art wurde die Flüssigkeit, anstatt mit Salzsäure, mit Salpetersüure angesäuert und bis gegen $240^{\circ}$ erhitzt. Hierdurch hatten sich einzelne, aber sehr deutliche Hexaëder gebildet, doch auch zugleich wieder solche prismatische 


\section{Scheerer $\mathfrak{u}$. Drechsel: Künstliche Darstellung}

spiessige Kryställchen: ${ }^{3}$ Wegen des untergeordneten Interesses, welches das Fluorbaryum für nus hatte, setzten wir die Versuche nit demselben nicht weiter fort.

Schwefelsaurer Baryt zeigt, trotz seiner ausserordentlichen Schwerlöslichkeit in Wasser, grosse Neigung zum Krystallisiren auf nassem Wege; denn alle Niederschläge desselben seheinen aus mikroskopischen Krystallen zu. bostehen, oder sich wenigstens sehr bald darin umzuwandeln. Etwas grössere Krystalle erhält man durch Anwendung besserer Lösungsmittel. Als eines derselben wendete Mittentzwei ${ }^{2}$ ) ein Gemisch von salpetersaurem Ammoniak und Salzsïure an. Senarm on $t^{3}$ ) orhielt mikroskopische Schwerspathkrystalle durch Einwirkung einer. Lösung von kohlensaurem Natron auf frisch gefällten schwefelsauren Baryt bei einer Temperatur von $250^{\circ}$. Statt des kohlensauren Natrons diente ihm, mit ähnlichem Erfolg, auch Salzsäure. Mac $\left.e^{4}\right)$ wendete eine Methode an, der das Princip möglichst verlangsamter Krystallbildung zu Grumde lag. Er liess sehr verdünnte Iiösungen von salpetersanrem Baryt und Eisenvitriol durch eine besondere Vorrichtung äusserst langsam in einander fliessen, wodurch sich am Orte des Zusammenflusses nach und nach kleine Schwerspathkrystalle bildeten. Wenn auch dem Eisenvitriol hierbei andere schwefelsaure Salze vorzuziehen sein dïrften, so ist doch das Prinzip der Metbode das zweokmässigste zur Erzielung möglichst grosser Krystalle.")

1) Dass diese Gebilde Doppel-Verbindungen suien, dafür scheint auch oine weiter unten angeführte Beoluachtung zu sprechen.

2) Mieses Journ. 75, 214.

3) Ann. ch. phys. 32, 129.

4) Dieses Journ. 59, 367 .

b) Als eives besonders wirksanen Lösungsmittels für sohwetelsauren Baryt muss hier auch noch der einbasischen (Meta-) Phosphorsänre gedacht werden. Ich habe früher gezeigt, dass dieselbo unter gewissen Umstândon verhältnissmässig grosse Mengen von schwefelsanrem Baryt eufgelöst zu halten vermag. In dem Maasse nun, als sich in solchen Lösungen dio oinbasisohe Phosphorsäure in droiba. sinche umsetzt - was äusearst langsam geschiøht - krystallisirt. scluwefelsaurer Baryt heraus.

s. 
Die durch höhere Temperatur gesteigerte Löslichkeit des schwefelsauren Baryts in Wasser kann ebenfalls zur Krystallbildung desselben benutzt werden. Eine stark verdünnte Chlorbaryumlösung mit etwas übersohügsiger Schwefelsäure versetzt und wẩhrend 12 Stunden bis auf $245^{\circ}$ erhitzt, gab bedeutend grössere - theilweise zu Gruppen verwachsene - Krystalle, als sich durch Fällung unter gewöhnlichen Umständen bildeten. Dies zeigte nicht blos die mikroskopische Untersuchung, sondern ging zugleich daraus hervor, dass, während eine bei gewöhnlicher T'emperatur gefällte Iösung nach dem Umrühren $1 \frac{1}{2}$ bis 2 Stunden zu ihrer Klärung brauchte, dies in einer bei $245^{\circ}$ behandelten Flilssigkeit schon nach 3 bis 5 Minuten erfolgte. Die auf letztere Art gebildeten Krystalle hatten sich nicht an der Röhrenwandung festgesetzt, oder waren wenigstens sehr leicht davon zu entfernen.

Von besonderem Interesse erschien es, da Flussspath und Schwerspath in Gängen so of neben einander vorkommen, solche nachbarlichen Gebilde künstlich hervorzurufen. In dieser Absicht wurden die folgenden Versuche von uns engestellt.

Fluorcalcium und schwefelsarer Baryt. Es liess sich vermuthen, dass sehwefelsaurer Kalk (Gyps) und Fluorbaryum unter gewissen Verhältnissen zersetzend auf einander einwirken würden, und dass dadurch sohwefelsaurer Baryt (Schwerspath) und Fluorealeium (Flussspath) entstehen müssten.

Demzufolge wurde zunächst ein Gemenge ron 1 Aequivalent Fluorbaryum und 1 Aequivalent wasserfreiem schwefelsaurem Kalk mit einer grösseren Quantität $\mathrm{K} \theta \mathrm{l}+\mathrm{Na \theta l}$ im Platintiegel zusammengeschmolzen. Nach Behandlung der Schmelze mit Wasser blieb cin krystallinisches Pulver ungelöst, in welchem das bewaffnete Auge meist nadolförmige Gebilde gewahrte, aber nichts was anf tesserale Gestaltung bezogen werden konnte. Die Nädelchen erwiesen sich unter dem Mikroskop im polarisirten Lichte als optiseh zweiaxig. Fine Wiederholung dieses Versnchs modificirten wir auf folgende Weise. In zwei Platintiegeln 


\section{Scheerer a. Drechsel: Künstliche Darstellung}

wurde $\mathrm{KGl}+\mathrm{NaGl}$ zum klaren Fluss eingeschmolzen, und darauf in den einen etwas Fluorbaryum, in den andern die äquivalente Menge schwefelsauren Kalkes geschïttet. Nachdem sich dieselben klar aufgelöst hatten, wurden beide glühend füssige Massen mit einander vermischt. Sofort entstand in dem heissflïssigen Gemisoh ein sich allmälig senkender Niederschlag. Nach Auslaugung der langsam erstarrten Schmelze vermochten wir in dem krystallinischen Rückstande nichts wesentlich anderes zu erkennen als beim ersten Versuch. Auch diesmal hatte es wieder den Anschein, dass nioht zweierlei Gebilde entstanden waren, sondern sich nur ein chemisches Produkt gebildet hatte. Die nähere Untersuchung desselben ergab schwefeleauren Baryt und Fluorcalcium in gleichen Aequivalenten.

Durch Anwendung des nassen Weges hofften wir zu einem unzweideutigeren Resultate zu gelangen. In eine unten zugeschmolzene Glasröhre wurde etwas Fluorbaryum geschüttet, durch Salzsäure angesäuertes Wasser daraufgegossen und dann ein Stück krystallisirter Gyps (Marienglas) mittelst eines Platindrahtes and Platinblechs so angebracht, dass dasselbe möglichst entfernt vom Fluorbaryum gehalten wurde. In der nun anch an dem anderen Ende zugeschmolzenen und horizontal in den KanonenApparat gelegten Glasröhre befanden sich also am oinen Ende Fluorbaryum und am andern Ende Gyps, beide unter der Wasserschicht. Nach lOstündigem Frhitzen bis anf etwas iiber $240^{\circ}$ and langsamer Abküblung, waren überaus zahlreiche prismatische Krystalle entstanden; nirgends aber liess sich eine Spur vor tesseralen Gebilden entdecken. Soviel stand also jedenfalls fest, dass hierbei kein Flussspath erzeugt worden war. Die prismatischen Krystalle besassen verschiedenen Habitus, und zwar liessen sich folgende drei Arten leicht von einander unterscheiden. 1) Lange sechsflächige Prismen (zum Theil über $1 \mathrm{Mm}$. lang bei nur 0,005 bis $0,01 \mathrm{Mm}$. Dicke), an denen mitunter eine dachförmige Zuspitzung deutlich bemerkbar. Sie ergaben sich bei näherer Prufung gls Gypskrystalle. 
Durch längeres Behandeln mit salzsïurehaltigem Wasser wurden sie allmählich vollständig gelöst, während die beiden andern Arten der Krystalle unverändert zurückblieben. 2) Kurze Prismen (meist von ungefähr 0,03 Mm. Länge bei $0,01 \mathrm{Mm}$. Durchmesser), welche sich als rhombische Krystalle von der Form $\infty$ P.oP. $\infty$ P $\infty$ (nach Naumann's Bezeichnung) auffassen liessen. Ihre grösste Dimension befand sich in der Richtung der Makrodiagonale. Dieser ganze Habitus unterstützt die Vermuthung, dass es Anhydritkrystalle ${ }^{1}$ ) waren, dann freilich auynahmsweise ohne die fast stets an denselben auftretenden Flächen $\infty \breve{P} \infty$. 3) Kleine nadelförmig spiessige Kry. stalle (von $0,03 \mathrm{Mm}$. Liange bei sehr geringem, oft kaum $0,001 \mathrm{Mm}$. betragendem Durchmesser). Da es nicht mög. lich war, diese letzteren beiden Arten der Krystalle von einander zu sondern, so konnte nur das Gemenge derselben chemisch untersucht werden. Dabei ergaben sich wieder Schwefelsäure, Flusssäure, Baryterde und Kalkerde als Bestandtheile.

Obwohl es sich hiernach zu bestätigen schien, dass eine chemische Doppel-Verbindung von schwefelsaurem Baryt und Fluorcalcium, nicht aber jedes dieser Salze für sich, gebildet worden war, wünschten wir diese befremdende Thatsache noch unzweifelhafter darzulegen. Dies konnte vermittelat Anwendung des Princips der verlang. samten Krystallbildung geschehen, wodarch wir grössere Krystalle jener fraglichen Verbindung zu erhalten hofften. Allerdings musste dabei auf die Mitwirkung einer höhern Temperatur verzichtet worden.

Eine U-förmig gebogene Glasröhre, an welcher das Mittelstück verhältnissmässig lang und von grossem Durch.

1) Gelang jedoch nicht solche Krystalle zu orhalten, als Ma. rienglas für sich unter Wasser bis auf $250^{\circ}$ erhitzt wurde. Hierbei bildeten sich blos jene langen Gypskrystalle, und das nicht geloste Marienglas zeigte sich in eine faserig krystallinische Masse umgo wandelt. Es seheint demnach für die Bildung der anhydritartigen Krystallo die Gegenwart des Fluorbaryums in irgend einer Woise wesentlich zu sein. 


\section{Scheerer u. Drechsel: Künstliche Varstellung}

messer war, wurde mit Wasser gefüllt und an ihrem einen Finde mit Fluorbaryum, an ihrem anderen Ende mit Gyps beschickt; derartig; dass diese von Filtrirpapier umhüllten Salze lockere Pfropfe in beiden Röhrenschenkeln bildeten. Das ins Wasser eintauchende Filtrirpapier bewirkte das Fenchtwerden und allmälige Auflösen der Salze. Die so gebildeten Lösungen senkten sich in den lothrecht stehenden Röhrensehenkeln und trafen im Mittelpunkt sehr langsam und verdünnt zusammen. Während wochenlanger Zeit setzten sich an der Röhrenwandung beträøhtliche Mengren von Krystallen ab, zu rehr oder weniger grossen Gruppen vereint, alle aber - wegen der geringeren Löslichkeit des Fluorbaryums im Vergleich mit der des Gypses - beträchtlich näher an der Lösungsquelle des erstgenannten Salzes als an der des zweiton. Als diese (in Wasser völlig unlöslichen) Krystallgebilde unter dem Mikroskop betraohtet wurden, boten sie einen durchaus anderen Anblick dar, als die des vorbeschriebenen Versuchs: Weder nadelförmige noch andere prismatische Krystalle waren bemorkbar. Dagegen zeigten sich iiberaus zahlreiche Krystallskelette - viele von schönster Tannenbaumform - mit schiefwinklig angelegten Zweigen und mit Zuspitzungen, die auf keine tesserale Gestalt zu beziehen waren. Ferner gab es tafelförmige Gestalten, und darunter von einer beilförmigen Art, wie sie für gewisse Schwerspäthe charakteristisch ist. Alle diese Gebilde gingen durch $Z$ wischenstufen in einander über, gehörten also einer und derselben Substanz an. Dass diese als Schwerspath in Auspruch za nehmen sei, erscheint um so unbedenklicher, als sich zugleich unzweifelhafte Flussspath-Krystalle, (mit Kantenlängen bis zu 0,04 Mm.) gebildet hatten; fast alle in scharfkantigster $\mathrm{Hexaëder-Form,} \mathrm{nur} \mathrm{wenige}$ mit zweifelhaften oktaëdrischen and rhombendodeksëdrischen Abstumpfungen. Meist waren sio den Schwerspathkrystallen aufgewachsen, zum Theil sassen sie auch einzeln und in kleinen Gruppen in der Nähe derselben. Bei gesteigerter Vergrösserung (bis zurn 300farhen) zeigten sich manche Schwerspathkrystalle - tannenbaum- 
ăhnliche Grebilde wie beilförmige 'Tafeln - ganz übersaiet von unregelmässig darüber ausgestreuten kleinen Hexaëdern Dio chemisohe Analyse dieses Krystall-Gemenges ergab, wie vorauszusehen, schwefelsauren Baryt und Fluorcalcium als alleinige Bestandtheile.

Obgleich unser augestrebter Hauptzweek - Flussspath und Schwerspath, als Produkte eines einfacben chemischen Processes neben einander darzustellen - durch das zulotzt erhaltene Resultat erreicht war, glaubten wir dasselbe doch durch eine Wiederholung des Versuchs feststellen zu müssen. Dieser zweite Versuch, von mehrwöchentlicher und längerer Dauer als der erste, bestätigte nicht nur vollkommen unsere früheren Beabachtungen, sondern lieferte uns auch noch grössere Flussspath-Hexaëder mit Kantenlängen bis zu $0,06 \mathrm{Mm}$. Zugleich hatten sich mehr tafelförmige Schwerspathkrystalle gebildet. -

Jene unerwartete Wahrnehmung: lass BaF und CaS bei hölerer lemperatur - und damit verbundener schnellerer Einwirkung - einander nicht zu Bä̈ und CaF in getrennter Gestalt zersetzen, sondern mit einander in chemische Verbindung treten, dürfte mit der bekannten Erfahrung zusammenhängen, dass Schwerspath und Flussspath zu einer leichtflïssigen Masse zusammenschmelzen. Man sollte jedoch meinen, dass, wenn eine chemische Verbindung $\dot{\mathrm{B}} \overrightarrow{\mathrm{S}}+\mathrm{Ca}$ wirklich existirt, sie auch in der Natur vorkommen müsste. Ein Mineral von dieser Zusammensetzung ist früher von Smith s on ') beobachtot, später aber von mehreren Mineralogen als blosses Gemenge betrachtet worden. Es bildete, als anscheinend derbe dichte Masse von 3,75 spec. Gew., eine etwa zolldicke Lage in einem Kalkschiefer von Derbyshire, begleitet von Kalkspath und Bleiglanz. Von einigen mineralogischen Lehrbüchern wird es als Fluorbaryt und Flussbaryt. (Baryto-Flnate of Lime, Thomson) angeführt. Ks wäre uns ron Interesse gewesen, dieses Mineral nïher zu unter-

1) Sehwoigger's Jahrbuch der Chom. und Phys., Jahrgang 1821, S. 362. Die Originalnotiz in Thowson's Annals of Philosophy 1820 , 


\section{Scheerer u. Drechsel: Künstliche Darstellung}

suchen; doch in den uns zu Gebote stehenden Sammlungen war es nicht vorhanden. Unsere Vermuthung, dass vielleicht auch manche faserige und stänglige Schwerspäthe flussspathhaltig sein könnten, bestätigte sich nicht. Weder im Bologneserspath und Freiberger Stangenspath, noch in verschiedenen faserigen Schwerspäthen der Freiberger Gegend und anderer Fundorte konnte ein Gehalt von Fluorcalcium entdeckt worden. Da Smith s on angiebt, dass der Fluobaryt in seinem Ausseren einem Kalkstein gleiche, so wurden auch noch einige derbe Schwerspäthe untersucht, so namentlich von Cobenstein, Steiermark, Baiern and einem unbekannten Fundorte. Aber auch in diesen. wie in einem erdigen Schwerspath von Borna, liess sich kein irgend erheblicher Fluorgehalt nachweisen. Ebensowenig konnte ein Gehalt von schwefelsaurem Baryt im schiefrigen Flussspath von Kemlos in Baiern und in dem derben Flussspath (Flusstein) von Strassberg am Harz erkannt werden. Einen fast gleichen negativen Erfolg gaben einige erdige Flussspäthe (Flusserden). Doch dürfte es von Interesse sein, hier anzufübren, dass die violblaue Flnsserde von Marienberg in Sachsen aus krystallinischen Partikeln besteht. unter denen sich zahlreiche Hexä̈der (bis zu $0,06 \mathrm{Mm}$. Kantenlänge) befinden. Ferner wurden in einer weissen Flusserde von unbekanntem Fundorte (meist aus mikroskopischen, runden Gebilden bestehend, mit theilweisen Andeutungen von Hexaëderform) vereinzelte flach-prismatisehe Kryställchen bemerkt, welche Schwerspath zu sein schienen. Die chemiche Prüfung dieser Flusserde ergab in der That einen kleinen Gehalt sowohl von Baryterde als Schwefelsäure. - Sämmtliche Schwerspathund Flussspath-Arten wurden der hiesigen bergakademischen Mineraliensammlung entlehnt. ${ }^{1)}$

Nach diesen Erfahrungen lässt sich annehmen, dass der Fluobaryt - wenn eine solche Mineralspecies wirklich existirt - nur an wenigen Orten anzutreffen sein

1) Durch die stets bereitwillige Güte meines Collegen Prof. Dr. Weis bach, dem ioh dafür meinen besten Dank sage. 
Fleischer: Ueber eine sehr einfache, rasch etc. 75 dürfte; und zwar aus dem Grunde, weil diese - wirkliche oder anscheinende - chemische Verbindung $\dot{\mathrm{BaS}}+\mathrm{CaF}$ sich blos unter Umständen bildet, wie sie in dem langsamen Entwicklungsgange der Natur am seltensten vorzukommen pflegen. Durch langsame gegenseitige Einwirkung verdünnter Solutionen von $\mathrm{BaF}$ und Cas kann, wie wir saheu, der Fluobaryt nicht entstanden sein; denn hierbei entstehen Schwerspath und Flussspath als getirennte Gebilde. Immerhin aber ist es möglich, dass an einigen Orten ausnahmsweise eine schnellere chemische Einwirkung stattgefunden haben mag.

In Betreff des verschiedenartigen krystallinischen Typus der von uns dargestellten Flussspathkrystalle verdient es schliesslich als ein Erfahrungsresultat hervorgehoben zu werden: dass langsame Entstehung, in Verbindung mit niederer Temperatur, die Hexa ëder-Bildung begünstigt, während schnelle Entstehung, in Verbindung mit hoher Temperatur, auf Oktaëder-Bildung hinwirkt.

Ueber eine sehr einfache, rasch arbeitende Filtrirvorrichtung nach Bunsen'schem Princip;

von

Dr. E. Fleisoher.

Das Bunsen'sche Filter hat hei allen Vortheilen der Zeitersparniss doch auch einige Nachtheile.

Bringt man z. B. Ceinkörnige Niederschläge auf ein solches Filter und arbeitet mit starker Luftverdünnung, so gehen dieselben vernöge des auf der Filterfäche lastenden Druckes leicht durch das Filter-Medium und trüben das Fitrat.

Ein anderer öfter eintretender Uebelstand liegt in dem Zerreissen des Filters an den den Platinrand berührenden 